\title{
TRIỂN KHAI HIỆ QUẢ BÀI TOÁN HIỆU CHỈNH CÁC HỆ SỐ ĐIỀU HOÀ CẦU CỦA MÔ HÌNH TRỌNG TRƯờnG TRÁI ĐẤT THEO THUẬT TOÁN COLOMBO O.L.
}

\author{
PGS. TSKH. HÀ MINH HOÀ \\ ThS. NGUYẼ̃N TUÂN ANH \\ Viện Khoa học Đo đạc và Bản đồ
}

\section{Tóm tắt:}

Sự phức tạp của việc giải quyết bài toán hiệu chỉnh các hệ số khai triển điều hòa cầu của mô hình trọng trường Trái đất EGM dựa trên cơ sở dư liệu dị thường trọng lực quốc gia nằm ở chỗ quá trình hiệu chỉnh tất cả các hệ số khai triển điều hòa cầu của mô hình trọng trường Trái đất EGM được lặp lại theo các vĩ độ trắc địa lần lượt từ nhỏ đến lớn của các đỉnh của các ô chuẩn dị thường trọng lực. Điều này làm phức tạp hóa việc tổ chức tính toán các hàm Legendre kết hợp được chuẩn hóa và làm tăng thời gian tính toán. Bài báo khoa học này đề xuất các biện pháp tổ chức tính toán hiệu quả để giải quyết bài toán nêu trên.

\section{1. Đặt vấn đề}

Việc tính toán dị thường độ cao dựa trên các dữ liệu dị thường trọng lực mặt đất được xác định trên lãnh thổ quốc gia và dữ liệu dị thường trọng lực toàn cầu được xác định từ mô hình trọng trường Trái đất EGM theo tích phân Stokes luôn là bài toán nan giải đối với các quốc gia có diện tích không lớn như Việt Nam. Theo tài liệu (Featherstone W.E., Kirby J.F., Hirt C., Filmer M.S., Claessens S.J., Brown N., Hu G., Johnston (2011)), để làm điều này, chúng ta cần các dữ liệu dị thường trọng lực mặt đất với bán kính từ $2^{\circ} 5$ đến $5^{\circ}$ xung quanh điểm tính toán dị thường độ cao bất kỳ trên lãnh thổ quốc gia. Điều này không thực tế khi đặt ra vấn đề thu thập các dữ liệu trọng lực tại các quốc gia lân cận.

Khi tính đến một thực tế rằng các dư liệu dị thường trọng lực của các quốc gia và các tổ chức quốc tế trên phạm vi toàn cầu đã được thu thập và được sử dụng để tính toán các hệ số khai triển điều hòa cầu của mô hình EGM, chúng ta chỉ cần sử dụng các dữ liệu dị thường trọng lực mới được xác định trên lãnh thổ quốc gia để hiệu chỉnh các hệ số khai triển điều hòa cầu của mô hình EGM sao cho phù hợp với trọng trường Trái đất trên lãnh thổ quốc gia, thêm vào đó các dữ liệu dị thường trọng lực mới này chưa được các tổ chức quốc tế sử dụng để tính toán các hệ số khai triển điều hòa cầu của mô hình $E G M$. Việc hiệu chỉnh các hệ số khai triển điều hòa cầu của mô hình EGM cho phép sử dụng mô hình EGM được hiệu chỉnh để tính toán một cách tin cậy các đại lượng vật lý như dị thường độ cao, gia tốc lực trọng trường, các thành phần độ lệch dây dọi với độ chính xác cao tại mọi điểm trên lãnh thổ quốc gia. Cách tiếp cận này với mục đích khai thác cơ sở dữ liệu (CSDL) dị thường trọng lực trên lãnh thổ Việt Nam đã được đề xuất, nghiên cứu trong các tài liệu (Hà Minh Hoà (2014); Hà Minh Hoà, Nguyễn Tuấn Anh (2014)).

Trong tài liệu (Hà Minh Hoà (2014)) đã luận chứng cho cơ sở khoa học của phương pháp cầu phương do Colombo O.L. đề xuất vào năm 1981 trong tài liệu (Colombo O.L. (1981)) để hiệu chỉnh các hệ số khai triển điều hòa cầu của mô hình EGM. Trong tài liệu 
(Hà Minh Hoà, Nguyễn Tuấn Anh (2014)) đã công bố kết quả thực nghiệm thuật toán Colombo O. L. trên khu vực miền Bắc Việt Nam với 5000 giá trị dị thường trọng lực.

Trong tài liệu (Nguyễn Tuấn Anh (2013)) đã nghiên cứu sự biến thiên của các giá dị thường độ cao trên lãnh thổ Việt Nam theo bậc khai triển của mô hình EGM2008 và đã xác định được rằng dị thường độ cao được tính toán sẽ thay đổi khi bậc $\mathrm{n}$ thay đổi từ 181 đến 2159. Điều này cũng dễ hiểu, bởi vì theo tài liệu (Hirt C., Marti U., Brki B. and Featherstone W.E. (2010)), các hệ số khai triển điều hoà của mô hình EGM2008 ở mức giữa 181 - 2159 được xác định từ các giá trị dị thường trọng lực mặt đất trong các ô chuẩn $5^{\prime} \times 5^{\prime}$ trên phạm vi toàn cầu. Như vậy, khi đặt ra bài toán hiệu chỉnh các hệ số khai triển điều hòa cầu của mô hình EGM2008 dựa trên các dữ liệu dị thường trọng lực trên lãnh thổ Việt Nam, các hệ số khai triển điều hòa cầu cần hiệu chỉnh có số lượng rất lớn (hơn 4 triệu hệ số). Do đó, khi giải quyết bài toán được đặt ra đối với mô hình EGM2008, chúng ta sẽ hiệu chỉnh các hệ số khai triển điều hòa cầu từ bậc $\mathrm{n}_{\min }=181$ đến bậc $\mathrm{n}_{\max }=2159$.

Với mục đích tăng nhanh thời gian tính toán khi giải quyết bài toán nêu trên, bài báo khoa học này sẽ đề xuất sơ đồ triển khai hiệu quả quá trình tính toán theo thuật toán Colombo O. L.

\section{Giải quyết vấn đề}

Chúng ta trình bày lại thuật toán Colombo O.L. được trình bày trong tài liệu (Hà Minh Hoà (2014)). Trong CSDL dị thường trọng lực, các ô chuẩn với kích thước $\Delta B x \Delta L$, thường là $3^{\prime}$ x 3' hoặc $5^{\prime} \times 5^{\prime}$, được phân bố với $\mathrm{M}$ ô chuẩn theo vĩ tuyến và $\mathrm{N}$ ô chuẩn theo kinh tuyến. Chúng ta sắp xếp thứ tự của các ô chuẩn dị thường trọng lực được đưa vào tính toán theo thứ tự tăng dần của vĩ độ trắc địa và kinh độ trắc địa của đỉnh cơ sở của mỗi ô chuẩn. Khi ký hiệu $(\mathrm{i}, \mathrm{j})$ là số hiệu của ô chuẩn, đỉnh cơ sở của ô chuẩn (i,j) là đỉnh có đồng thời vĩ độ trắc địa $B_{i}$ và kinh độ trắc địa $L_{j}$ không lớn hơn so với các tọa độ trắc địa của 3 đỉnh còn lại. Khi đó, đối với toàn bộ $C S D L$ dị thường trọng lực, các chỉ số $i=1,2, \ldots, N$ và $j$ $=1,2, \ldots, \mathrm{M}$. Các hệ số khai triển điều hòa cầu được hiệu chỉnh theo công thức:

$$
\widetilde{\bar{C}}_{n, m}=\bar{C}_{n, m}+\delta \bar{C}_{n, m} ; \quad \widetilde{\bar{S}}_{n, m}=\bar{S}_{n, m}+\delta \bar{S}_{n, m} \text {, }
$$

ở đây $\bar{C}_{n, m}, \bar{S}_{n, m}$ là các hệ số điều hòa cầu của mô hình EGM, các số hiệu chỉnh được tính theo công thức sau:

$$
\left\{\begin{array}{l}
\delta \bar{C}_{n, m} \\
\delta \bar{S}_{n, m}
\end{array}\right\}=K_{n} \cdot \sum_{i=1}^{N} \bar{\chi}_{n, m}^{i} \cdot \sum_{j=1}^{M} \delta \Delta \bar{g}(i, j) \cdot\left[\left\{\begin{array}{l}
A(m) \\
-B(m)
\end{array}\right\} \cdot \cos m L_{j}+\left\{\begin{array}{l}
B(m) \\
A(m)
\end{array}\right\} \cdot \sin m L_{j}\right] .
$$

Trong công thức (2) $\quad K_{n}=\frac{a_{e}^{2}}{4 \pi \cdot G \cdot M \cdot(n-1)}$

$$
\begin{aligned}
& \bar{\chi}_{n, m}^{i}=\left(\frac{\rho_{i}}{a_{e}}\right)^{n} \cdot \frac{\bar{P}_{n, m}\left(\sin B_{i}\right) \cdot \Delta \sigma_{i, j}}{q_{n}}, \\
& q_{n}=\left\{\begin{array}{lll}
\beta_{n}^{2} & \text { khi } & 0 \leq n \leq n_{\max } / 3, \\
\beta_{n} & \text { khi } & n_{\max } / 3<n \leq n_{\max }, \\
1 & \text { khi } & n_{\max }<n .
\end{array}\right.
\end{aligned}
$$




\section{Nghiên cúu}

$\beta_{n}$ - tham số Pellinen L.P. được tính theo công thức Pellinen L.P hoặc công thức Meisl P. hoặc công thức Sjoberg L. E. (xem chi tiết trong Hà Minh Hoà (2014)), $n_{\max }$ - bậc khai triển cực đại của mô hình EGM (đối với mô hình EGM2008 $n_{\max }=2159$, diện tích ô chuẩn $(\mathrm{i}, \mathrm{j})$ được tính theo công thức: $\quad \Delta \sigma_{i j}=\Delta L$. $\left[\sin B_{i}-\sin \left(B_{i}+\Delta B\right)\right]$,

các hệ số

$$
\begin{gathered}
A(m)=\left\{\begin{array}{lll}
(\sin m \cdot \Delta L) / m \cdot \Delta L & k h i & m \neq 0, \\
1 & k h i & m=0,
\end{array}\right. \\
B(m)=\left\{\begin{array}{lll}
(\cos m \cdot \Delta L-1) / m \cdot \Delta L & k h i & m \neq 0, \\
0 & \text { khi } & m=0,
\end{array}\right.
\end{gathered}
$$

Trong các công thức (2), (7) chúng ta phải tính các giá trị $\cos (\mathrm{m} X)$ và $\sin (\mathrm{m} X)$ với biến $X$ bất kỳ. Do đó, việc đầu tiên là cần xây dựng các công thức truy hồi để tính toán các giá trị này. Chúng ta có:

$$
\cos (m \cdot X)=\cos [(m-1) X+X]=\cos [(m-1) X] \cdot \cos X-\sin [(m-1) \cdot X] \cdot \sin X .
$$

Mặt khác $\sin [(m-1) \cdot X]=\sin [(m-2) X+X]=\sin [(m-2) X] \cdot \cos X+\cos [(m-2) \cdot X] \cdot \sin X$,

$$
\begin{aligned}
& \sin [(m-1) \cdot X] \cdot \sin X=\sin [(m-2) X] \cdot \sin X \cdot \cos X+\cos [(m-2) \cdot X] \cdot \sin ^{2} X= \\
& =\sin [(m-2) X] \cdot \sin X \cdot \cos X+\cos [(m-2) \cdot X]-\cos [(m-2) \cdot X] \cdot \cos ^{2} X= \\
& =\cos X \cdot\{\sin [(m-2) X] \cdot \sin X-\cos [(m-2) \cdot X] \cdot \cos X\}+\cos [(m-2) \cdot X]= \\
& =-\cos X \cdot \cos [(m-1) \cdot X]+\cos [(m-2) \cdot X]
\end{aligned}
$$

nên công thức truy hồi để tính toán giá trị $\cos (\mathrm{mX})$ có dạng:

$$
\cos (m X)=2 \cdot \cos X \cdot \cos [(m-1) \cdot X]-\cos [(m-2) \cdot X]
$$

Tương tự, chúng ta có:

$$
\sin (m \cdot X)=\sin [(m-1) \cdot X+X]=\sin [(m-1) \cdot X] \cdot \cos X+\cos [(m-1) \cdot X] \cdot \sin X .
$$

Mặt khác $\quad \cos [(m-1) \cdot X]=\cos [(m-2) \cdot X+X]=\cos [(m-2) \cdot X] \cdot \cos X-\sin [(m-2) \cdot X] \cdot \sin X$,

$$
\begin{aligned}
& \cos [(m-1) \cdot X] \cdot \sin X=\cos [(m-2) \cdot X] \cdot \sin X \cdot \cos X-\sin [(m-2) \cdot X] \cdot \sin ^{2} X= \\
& =\cos [(m-2) \cdot X] \cdot \sin X \cdot \cos X-\sin [(m-2) \cdot X]+\sin [(m-2) \cdot X] \cdot \cos ^{2} X= \\
& =\cos X \cdot\{\cos [(m-2) \cdot X] \cdot \sin X+\sin [(m-2) \cdot X] \cdot \cos X\}-\sin [(m-2) \cdot X]= \\
& =\cos X \cdot \sin [(m-1) \cdot X]-\sin [(m-2) \cdot X]
\end{aligned}
$$

Cuối cùng $\sin (m \cdot X)=2 \cdot \cos X \cdot \sin [(m-1) \cdot X]-\sin [(m-2) \cdot X]$

Chúng ta cũng cần thiết chỉ ra rằng các công thức truy hồi (8), (9) đã được Rapp R.H. sử dụng trong phần mềm tính toán độ cao geoid từ mô hình EGM96 được lập theo ngôn ngữ lập trình Fortran77.

Trong công thức (2) giá trị $\delta \Delta \bar{g}(i, j)=\overline{\Delta g(i, j)}-\Delta g_{E G M}(i, j)$,

ở đây $\overline{\Delta g(i, j)}$ là giá trị trung bình của dị thường trọng lực của ô chuẩn (i,j) được tính 
toán tại điểm trọng tâm với vĩ độ trung bình $\bar{B}(i, j)$ và kinh độ trung bình $\bar{L}(i, j)$ từ các giá trị dị thường trọng lực tại 4 đỉnh của ô chuẩn theo công thức nội suy song tuyến Jurkin I.G. - Neyman lu. M. (xem chi tiết trong Hà Minh Hoà (2014)), còn giá trị $\Delta g_{E G M}(i, j)$ tại điểm trọng tâm được xác định từ mô hình EGM.

Để tổ chức tính toán hiệu quả các số hiệu chỉnh của các hệ số khai triển điều hòa cầu theo công thức (2), chúng ta biểu diễn lại công thức (2) dưới dạng:

$$
\left\{\begin{array}{l}
\bar{C}_{n, m} \\
\bar{\delta}_{n, m}
\end{array}\right\}=K_{n} \cdot \sum_{i=1}^{N} \bar{\chi}_{n, m}^{i} \cdot H_{i}(m),
$$

ở đây vectơ $H_{i}$ cột kích thước $2 \times 1$ đối với mỗi hàng thứ $\mathrm{i}(\mathrm{i}=1,2, \ldots, \mathrm{N})$ được xác định theo công thức:

$$
H_{i}(m)=\sum_{j=1}^{M} \delta \Delta \bar{g}(i, j) \cdot\left[\left\{\begin{array}{l}
A(m) \\
-B(m)
\end{array}\right\} \cdot \cos m L_{j}+\left\{\begin{array}{l}
B(m) \\
A(m)
\end{array}\right\} \cdot \sin m L_{j}\right] .
$$

Để tính toán tất cả $\mathrm{N}$ vectơ cột $H_{i}$ theo công thức (12), đầu tiên chúng ta xác định các giá trị $\delta \Delta \bar{g}(i, j)$ của tất cả các ô chuẩn trong CSDL dị thường trọng lực theo công thức (10) và sắp xếp theo hàng dưới dạng ma trận $\mathrm{Z}$ kích thước $\mathrm{N} \times \mathrm{M}$ như sau:

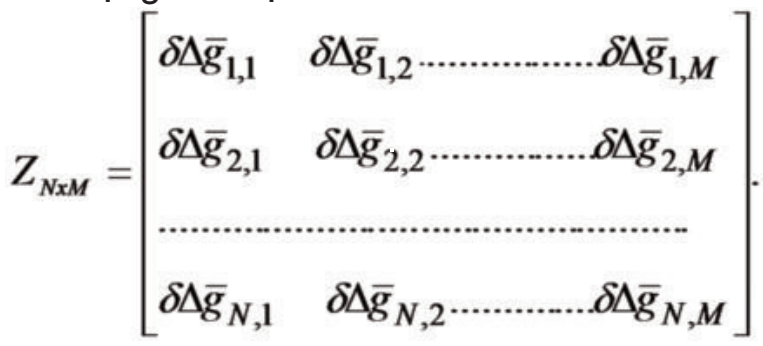

Tổng số $\left(n_{\max }+1\right)$ các cặp giá trị $\mathrm{A}(\mathrm{m}), \mathrm{B}(\mathrm{m})$ được tính toán với chỉ số $\mathrm{m}$ thay đổi từ 0 đến $n_{\text {max }}$. Đối với mô hình EGM2008 có tất cả 2160 cặp giá trị nêu trên. Để tiện sử dụng tiếp theo, chúng ta tổ chức file trực truy (tạm ký hiệu là file $A B$ ) với số thứ tự của bản ghi 1 đến $\left(n_{\max }+1\right)$. Như vậy, chỉ số $\mathrm{m}$ sẽ tương ứng với bản ghi $\mathrm{m}+1$. Sau khi tính toán được cặp hệ số $A(m), B(m)$, lần lượt theo $M$ kinh độ $L_{1}, L_{2}, \ldots, L_{M}$ của các điểm cơ sở tiến hành tính cặp giá trị $\cos m \cdot L_{j}, \sin m \cdot L_{j}(\mathrm{j}=1,2, \ldots, \mathrm{M})$ theo các công thức (8), (9) và tính tiếp hai giá trị: $T(m, j)=A(m) \cdot \cos m L_{j}+B(m) \cdot \sin m L_{j} ; U(m, j)=B(m) \cdot \cos m L_{j}+A(m) \cdot \sin m L_{j}$.

Hai giá trị trên được lưu lại trên cùng hàng $\mathrm{j}$ của bản ghi $m+1$. Như vậy trong bản ghi $m+1$ sẽ có $M$ hàng, mỗi hàng chứa hai giá trị $T(m, j)$ và $U(m, j), j=1,2, \ldots M$. Với cách tổ chức file trực truy $\mathrm{AB}$ như ở trên, khi tính toán các số hiệu chỉnh $\delta \bar{C}_{n, m} ; \delta \overline{\mathrm{S}}_{n, m}$, chúng ta chỉ việc gọi bản ghi $m+1$ ra và kết hợp với ma trận $Z_{\mathrm{NxM}}$ (13) dễ dàng tính toán vectơ cột $H_{i}(m)$ kích thước $2 \times 1$ theo công thức (12) đối với mỗi chỉ số $\mathrm{i}(\mathrm{i}=1,2, \ldots, \mathrm{N})$.

Trong các công thức (3) và (4), $a_{e}=6378137 m$ là bán kính bán trục lớn của ellipsoid WGS84 quốc tế, G.M - hằng số trọna trường địa tâm của Trái đất được sử dụng trong mô hình EGM, còn trong công thức (4) $\rho_{i}$ là bán kính địa tâm của đỉnh cơ sở thứ i với vĩ độ trắc địa $B_{i}$, thêm vào đó

$$
\rho_{i}=\sqrt{X_{i}^{2}+Y_{i}^{2}+Z_{i}^{2}},
$$


ở đây $X_{i}=N_{i} \cdot \cos B_{i} \cdot \cos L_{i}, Y_{i}=N_{i} \cdot \cos B_{i} \cdot \sin L_{i}, Z_{i}=N_{i} \cdot\left(1-e^{2}\right) \cdot \sin B_{i}$, bán kính cong của đường thẳng đứng thứ nhất của điểm đỉnh cơ sở thứ i được xác định theo công thức:

$$
N_{i}=\frac{a_{e}}{\sqrt{1-e^{2} \cdot \sin ^{2} B_{i}}}
$$

$e^{2}=0.0066943799013$ - tâm sai thứ nhất của ellipsoid WGS84 quốc tế.

Việc tính toán hệ số $\bar{\chi}_{n, m}$ theo các công thức (4), (5) tương đối phức tạp. Việc tính diện tích ô chuẩn $(i, j)$ theo công thức $(6)$ khá đơn giản khi chỉ cần biết vĩ độ trắc địa $B_{i}$ của đỉnh cơ sở của ô chuẩn này. Vấn đề phức tạp hơn là xác định hàm Legendre kết hợp được chuẩn hóa $\bar{P}_{n, m}\left(\sin B_{i}\right)$. Trong tài liệu (Hà Minh Hoà (2014)) đã trình bày các công thức xác định hàm Legendre được chuẩn hóa $\bar{P}_{n, m}(\sin B)$ như sau:

Khi $\mathrm{m}=0, \mathrm{n}=2, \ldots, \mathrm{n}_{\text {max }}: \bar{P}_{n, 0}(\sin B)=\sqrt{2 \cdot n+1} \cdot P_{n}(\sin B)$,

ở đây hàm Legendre $P_{n}(\sin B)$ được xác định theo công thức truy hồi:

$$
P_{n}(\sin B)=\frac{2 \cdot n-1}{n} \cdot \sin B \cdot P_{n-1}(\sin B)-\frac{n-1}{n} \cdot P_{n-2}(\sin B) \text {. }
$$

Các giá trị ban đâu được sử dụng đế tính toán theo công thức truy hồi bao gồm

Khi $m \neq 0, n=2, \ldots, n_{\max }$ :

$$
P_{0}(\sin B)=1, P_{1}(\sin B)=\sin B, P_{2}(\sin B)=\frac{3}{2} \cdot \sin ^{2} B-\frac{1}{2} \ldots
$$

$$
\bar{P}_{n, m}(\sin B)=\sqrt{2 \cdot(2 \cdot n+1) \cdot \frac{(n-m) !}{(\mathrm{n}+\mathrm{m}) !}} \cdot P_{n, m}(\sin B),
$$

ở đây hàm Legedre kết hợp $P_{n, m}(\sin B)$ được xác định theo công thức truy hồi:

với $\boldsymbol{m} \leq \boldsymbol{n}-2$.

$$
P_{n, m}(\sin B)=\frac{2 \cdot n-1}{n-m} \cdot \sin B \cdot P_{n-1, m}(\sin B)-\frac{n+m-1}{n-m} \cdot P_{n-2, m}(\sin B)
$$

Các giá trị ban đầu được sử dụng để tính toán theo công thức truy hồi bao gồm

$$
\begin{aligned}
& P_{1,1}(\sin B)=-\cos B, P_{2,1}(\sin B)=3 \cdot \sin B \cdot \cos B, P_{2,2}(\sin B)=3 \cdot \cos ^{2} B, \\
& P_{3,1}(\sin B)=\frac{3}{2} \cdot \sin ^{3} B-\frac{3}{2} \cdot \sin B, P_{3,2}(\sin B)=15 \cdot \cos ^{2} B \cdot \sin B, \\
& P_{3,3}(\sin B)=15 \cdot \cos ^{3} B .
\end{aligned}
$$

Đối với hàm Legendre $P_{n, m}(\sin B)$, khi $\mathrm{m}>\mathrm{n}: P_{n, m}(\sin B)=0$. Công thức (14) chỉ cho phép tính toán các hàm Legendre khi mức $m \leq n-2$. Ví dụ, khi $n=181$, chúng ta chỉ có thể tính toán được hàm Legendre tối đa đến mức $m=179$. Đối với hàm Legendre có mức $\mathrm{m}=\mathrm{n}-1$, chúng ta xác định hàm $P_{n, n-1}(\sin B)$ theo công thức:

$$
P_{n, n-1}(\sin B)=(2 n-1) \cdot \sin B \cdot P_{n-1, n-1}(\sin B) \text {. }
$$

Đối với hàm Legendre có mức $\mathrm{m}=\mathrm{n}$, chúng ta xác định hàm $P_{n, n}(\sin B)$ theo công thức:

$$
P_{n, n}(\sin B)=-(2 n-1) \cdot \cos B \cdot P_{n-1, n-1}(\sin B) \text {. }
$$

Các công thức (14), (15), (16) cho phép xác định các hàm Legendre để giải quyết bài 
toán được đặt ra trong bài báo khoa học này.

Khi tính toán các số hiôll shỉnh $\delta \bar{C}_{n, m} ; \delta \bar{S}_{n, m}$ với các chỉ số $\mathrm{n}, \mathrm{m}$ xác định, chúng ta phải xác định $\mathrm{N}$ giá trị $\bar{\chi}_{n, m}^{i} \quad(\mathrm{i}=1,2, \ldots, \mathrm{N})$, thêm vào đó đối với mỗi chỉ số $\mathrm{i}$ chúng ta lại phải xác định xác định hàm Legendre kết hợp được chuẩn hóa $\bar{P}_{n, m}\left(\sin B_{i}\right)$ đối với đỉnh cơ sở của ô chuẩn có vĩ độ trắc địa $B_{i}$. Chính điều này đòi hỏi thời gian tính toán rất lớn khi tính toán các số hiệu chỉnh $\delta \bar{C}_{n, m} ; \quad \bar{S}_{n, m}$ với các chỉ số $\mathrm{n}, \mathrm{m}$ xác định theo công thức (11).

Để tăng nhanh thời gian tính toán, với vĩ độ trắc địa $\mathrm{B}$ xác định, chúng ta thấy rằng để xác định hàm Legendre kết hợp $P_{I, m}(\sin B)$ chúng ta cần biết trước hai đại lượng $P_{I-1, m}(\sin$ $B$ ) và $P_{I-2, m}(\sin B)$. Giả sử chúng ta tổ chức file trực truy (tạm ký hiệu là file $\mathrm{PL}$ ) với số thứ tự của bản ghi 1 đến $\left(n_{\max }+1\right)$. Như vậy, chỉ số $\mathrm{m}$ sẽ tương ứng với bản ghi $\mathrm{m}+1$. Trong mỗi bản ghi $\mathrm{m}+1$ chúng ta lưu giữ hai giá trị $P_{I-1, m}(\sin B)$ và $P_{I-2, m}(\sin B)$ với mục đích phục vụ việc tính toán hàm Legendre kết hợp $P_{l, m}(\sin B)$ theo các công thức (14), (15), (16). Sau khi đã tính toán được $P_{l, m}(\sin B)$, chúng ta lại lưu giữ trong bản ghi này hai giá trị $P_{l, m}(\sin$ $B)$ và $P_{I-1, m}(\sin B)$ để phục vụ việc tính toán tiếp theo hàm $P_{I+1, m}(\sin B)$ và cứ tiếp tục như vậy. Sơ đồ tính toán nêu trên chủ yếu được áp dụng khi chúng ta tính toán đồng thời các số hiệu chỉnh $\delta \bar{C}_{n, m} ; \quad \bar{S}_{n, m}$ có cùng bậc $\mathrm{n}$. Với bậc $\mathrm{n}$ nhỏ chỉ có một số bản ghi (tạm gọi là các bản ghi không rông) có chứa hai giá trị $P_{l, m}(\sin B)$ và $P_{l-1, m}(\sin B)$ còn các bản ghi còn lại rỗng. Khi bậc $n$ tăng dần, số bản ghi không rỗng sẽ tăng dần.

Với sơ đồ tính toán nêu trên, chúng ta tính toán các số hiệu chỉnh của các hệ số khai triển điều hòa cầu theo chế độ tích lũy. Khi đó công thức (11) được biểu diễn ở dạng sau:

$$
\begin{aligned}
& \left\{\begin{array}{l}
\delta \bar{C}_{n, m} \\
\delta \bar{S}_{n, m}
\end{array}\right\}=\left\{\begin{array}{l}
0.0 \\
0.0
\end{array}\right\}+K_{n} \cdot \bar{\chi}_{n, m}^{1} \cdot H_{1}(m)+K_{n} \cdot \bar{\chi}_{n, m}^{2} \cdot H_{2}(m)+\ldots+K_{n} \cdot \bar{\chi}_{n, m}^{i} \cdot H_{i}(m)+ \\
& +\ldots+K_{n} \cdot \bar{\chi}_{n, m}^{N} \cdot H_{N}(m) .
\end{aligned}
$$

Khi đó, với mỗi vĩ độ trắc địa $B_{i}$ của đỉnh cơ sở $(\mathrm{i}=1,2, \ldots, \mathrm{N})$, chúng ta tính toán được thành phần tương ứng $K_{n} \cdot \bar{\chi}_{n, m}^{l} \cdot H_{i}(m)$ trong công thức (17). Sau khi tính toán hiệu chỉnh tất cả các hệ số khai triên điêu hòa câu với $\mathrm{n}$ thay đổi từ $n_{\min }$ đến $n_{\max }$ và $\mathrm{m}$ thay đổi từ 0 đến $\mathrm{n}$ đối với mỗi vĩ độ trắc địa $B_{i}$, chúng ta tổ chức lại file trực truy $\mathrm{PL}$ cho vĩ độ trắc địa $B_{i+1}$ của đỉnh cơ sở tiếp theo. Tương tự, sau khi tính toán hiệu chỉnh tất cả các hệ số khai triển điều hòa cầu với bậc $n$ thay đổi từ $n_{\min }$ đến $n_{\max }$ và mức $m$ thay đổi từ 0 đến $n$ đối với mỗi vĩ độ trắc địa $B_{i+1}$, chúng ta tổ chức lại file trực truy $\mathrm{PL}$ cho vĩ độ trắc địa $B_{i+2}$ của đỉnh cơ sở tiếp theo và cứ tiếp tục như vậy cho đến vĩ độ trắc địa $\mathrm{B}_{\mathrm{N}}$.

Đối với CSDL dị thường trọng lực lớn, chúng ta phải chia CSDL này thành các phân khu nhỏ, thêm vào đó mỗi phân khu nhỏ được sử dụng để tính toán có $\mathrm{N}$ ô chuẩn dọc theo kinh tuyến và $\mathrm{M}$ ô chuẩn dọc theo vĩ tuyến. Cách chia $C S D L$ dị thường trọng lực thành các phân khu làm giảm nhẹ việc tổ chức tính toán trong phần mềm được xây dựng để hiệu chỉnh các hệ số điều hòa cầu của mô hình EGM dựa trên các dữ liệu dị thường trọng lực quốc gia.

Để đơn giản cho việc triển khai tính toán các số hiệu chỉnh $\delta \bar{C}_{n, m} ; \quad \bar{S}_{n, m}$ theo công 
thức (17) đối với mô hình EGM2008, đầu tiên chúng ta tạo ra file tuần tự (tạm gọi là file DCS) có cấu trúc như sau:

\begin{tabular}{|c|c|c|}
\hline 181 & 0 & 0.0 \\
\hline 181 & 1 & 0.0 \\
\hline 181 & 181 & 0.0 \\
\hline 182 & 0 & 0.0 \\
\hline 182 & 1 & 0.0 \\
\hline 2159 & 0 & 0.0 \\
\hline 2159 & 1 & 0.0 \\
\hline
\end{tabular}

Trong cấu trúc trên, cột 1 chứa chỉ số $\mathrm{n}$, cột 2 chứa chỉ số $\mathrm{m}$, cột 3 chứa giá trị $\delta \bar{C}_{n, m}$ và cột 4 chứa giá trị $\delta \bar{C}_{n, m}$. Sau khi tính toán xong đối với vĩ độ trẳc địa $\mathrm{B}_{1}$ của đỉnh cơ sở đầu tiên của các ô chuẩn trong CSDL dị thườna trona lực quốc gia cùng với việc tính toán tích lũy với các thành phần hiệu chỉnh $K_{n} \cdot \bar{\chi}_{n, m}^{1} \cdot H_{1}(m)$ theo công thức $(17)$, chúng ta lại chuyển file DCS về bản ghi đầu (rewind) và tiến hành tính toán đối với vĩ độ trắc địa $B_{2}$ của đỉnh cơ sở thứ hai và quá trình tính toán được lặp lại cho đến khi tính toán xong đối với vĩ độ trắc địa $\mathrm{B}_{\mathrm{N}}$ của đỉnh cơ sở cuối cùng. Sau đó tiến hành hiệu chỉnh các hệ số điều hòa cầu $\bar{C}_{n, m}, \bar{S}_{n, m}$ của mô hình EGM theo công thức (1).

\section{Kết luận}

Việc phân tích thuật toán của Colombo O. L. cho thấy đã hình thành hai nhóm dữ liệu. Nhóm thứ nhất bao gồm các thành phần thay đổi theo mức $m$ và các kinh độ trắc địa $L_{1}$, $\mathrm{L}_{2}, \ldots, \mathrm{L}_{\mathrm{M}}$ của các đỉnh cơ sở của các ô chuẩn dị thường trọng lực. Các thành phần của nhóm này được tổ chức tính toán trước cùng với ma trận các hiệu dị thường trọng lực mặt đất và toàn cầu. Nhóm thứ hai chủ yếu là các hàm Legendre được chuẩn hóa. Việc xử lý tính toán đối với các thành phần của nhóm thứ hai đòi hỏi thời gian tính toán rất lớn do để hiệu chỉnh các hệ số khai triển điều hòa $\bar{C}_{n, m}, \bar{S}_{n, m}$ với bậc $\mathrm{n}$ và hạng $\mathrm{m}$ xác định, chúng ta phải tính toán $\mathrm{N}$ lần các hàm Legendre được chuẩn hóa lần lượt theo $\mathrm{N}$ vĩ độ trắc địa $\mathrm{B}_{1}, \mathrm{~B}_{2}, \ldots, \mathrm{B}_{\mathrm{N}}$ của các đỉnh cơ sở của các ô chuẩn dị thường trọng lực.

Với mục đích giảm thời gian tính toán đối với các thành phần thuộc nhóm thứ hai, bài báo khoa học này đã đề xuất sơ đồ tính toán, theo đó đối với mỗi vĩ độ trắc địa $\mathrm{B}_{\mathrm{i}}$ ( $\mathrm{i}=$ $1,2, \ldots, N)$ chúng ta tiến hành hiệu chỉnh đồng thời tất cả các hệ số khai triển điều hòa với bậc $\mathrm{n}$ thay đổi từ $n_{\min }$ đến $n_{\max }$ và mức $\mathrm{m}$ thay đổi từ 0 đến $\mathrm{n}$. Khi đó, các hệ số điều hòa cầu được tính toán trong chế độ tích lũy. Bằng cách như vậy, chúng ta có thể đơn giản hóa 
việc tính toán các hàm Legendre theo công thức truy hồi khi sử dụng file trực truy để lưu giữ các hàm Legendre đã được tính toán ở các bước trước. Quá trình hiệu chỉnh đồng thời tất cả các hệ số khai triển điều hòa với bậc $\mathrm{n}$ thay đổi từ $n_{\min }$ đến $n_{\max }$ và mức $\mathrm{m}$ thay đổi từ 0 đến $\mathrm{n}$ theo chế độ tích lũy được thực hiện $\mathrm{N}$ lần lần lượt với từng vĩ độ trắc địa $\mathrm{B}_{\mathrm{i}}(\mathrm{i}=$ $1,2, \ldots, N) . O$

\section{Tài liệu tham khảo}

[1]. Colombo O.L. (1981). Numerical methods for harmonic analysis on the sphere. Scientific report No7, AFGL-TR-81-0038, The Ohio State University, Research Foundation, Columbus, Ohio 43212, $140 \mathrm{p}$.

[2]. Featherstone W.E., Kirby J.F., Hirt C., Filmer M.S., Claessens S.J., Brown N., Hu G., Johnston (2011). The AUSGeoid2009 model of the Australian Height Datum. Journal of Geodesy (online first), doi: 10.1007/s00190-010-0422-2.

[3]. Hà Minh Hoà (2014). Lý thuyết và thực tiễn của Trọng lực trắc địa. NXB Khoa học và Kỹ thuật, 592 trg., Hà Nội - 2014.

[4]. Hà Minh Hoà, Nguyễn Tuấn Anh (2014). Nghiên cứu khả năng hiệu chỉnh các hệ số khai triển điều hoà cầu của thế trọng trường Quả đất của mô hình EGM2008 dựa trên các dữ liệu đo trọng lực chi tiết ở Việt Nam. Báo cáo khoa học. Kỷ yếu Hội nghị Khoa học "Trắc địa và Bản đồ vì hội nhập quốc tế" ngày 08/07/2014. Viện Khoa học Đo đạc và Bản đồ, Hội Trắc địa, Bản đồ và Viễn thám Việt Nam, trg. 21 - 37.

[5]. Nguyễn Tuấn Anh (2013). Nghiên cứu xác định số bậc tối đa của mô hình hệ số điều hòa cầu trong thực tế tính toán dị thường độ cao trên lãnh thổ Việt Nam. Tạp chí Khoa học Đo đạc và Bản đồ, số 17, tháng 09/2013, trg. 5 - 9.0

\section{Summary}

\section{Effective Realization of task of correction for spherical harmonical coefficients of the Earth Gravitational Model by algorithm of Colombo O.L.}

\section{Assoc. Prof. Dr. Sc. Ha Minh Hoa}

\section{MSc. Nguyen Tuan Anh}

\section{Vietnam Institute of Geodesy and Cartography}

A complexity of solving a task of correction of the spherical harmonical coefficients of the Earth Gravitational Model EGM based on the state gravity anomaly database consists in that a process of the correction of the spherical harmonical coefficients of the Earth Gravitational Model EGM will be iterated by sequential change of the geodetic latitudes of points of grids of the the state gravity anomaly database from small value to biger. This problem complicates computational organization of normalized Legendre functions and increases computational time. This scientific article proposes means of effective computational organization for solving abovementioned task. $O$ 